\title{
Surgical perspective on treatment of pediatric undifferentiated sarcoma of the liver
}

\author{
Ana M. Calinescu ${ }^{1}$, Barbara E. Wildhaber ${ }^{1}$, Florent Guérin ${ }^{2}$ \\ 'Division of Pediatric Surgery, University Center of Pediatric Surgery of Western Switzerland, Geneva University Hospitals, \\ Geneva 1205, Switzerland. \\ ${ }^{2}$ Pediatric Surgery Unit, Bicêtre Hospital, Université Paris-Saclay, Paris 94275, France. \\ Correspondence to: Dr. Ana M. Calinescu, Division of Pediatric Surgery, University Center of Pediatric Surgery of Western \\ Switzerland, Geneva University Hospitals, 6 Rue Willy Donze, Geneva 1205, Switzerland. E-mail: ana-maria.calinescu@hcuge.ch
}

How to cite this article: Calinescu AM, Wildhaber BE, Guérin F. Surgical perspective on treatment of pediatric undifferentiated sarcoma of the liver. Hepatoma Res 2021;7:54. https://dx.doi.org/10.20517/2394-5079.2021.25

Received: 27 Feb 2021 First Decision: 12 Apr 2021 Revised: 13 May 2021 Accepted: 25 May 2021 First online: 31 May 2021

Academic Editors: Guang-Wen Cao, Piotr Czauderna Copy Editor: Yue-Yue Zhang Production Editor: Yue-Yue Zhang

\begin{abstract}
Surgical resection and chemotherapy are the mainstay of the treatment for undifferentiated embryonal sarcoma of the liver. Whether neoadjuvant chemotherapy should be systematically performed is a matter of debate; perioperative morbidity and mortality should be carefully weighed against chemotherapy-associated complications. In order to manage undifferentiated embryonal sarcoma of the liver and to allow for accurate outcome analysis, there is a clear need for standardization of disease extent as well as for a risk stratification system, including the PRETEXT grouping system, patient age, and tumor size.
\end{abstract}

Keywords: Undifferentiated embryonal sarcoma of the liver, staging system, positive margins, neoadjuvant chemotherapy

\section{INTRODUCTION}

Undifferentiated embryonal sarcoma of the liver (UESL) is the third most frequent liver malignancy in children. Its peak of incidence lays between that of hepatoblastoma (young children) and hepatocellular carcinoma (older children) $)^{[1]}$. Most children who develop UESL are aged 6 to 10 years ${ }^{[2]}$. The clinical presentation is often non-specific: an abdominal mass with or without abdominal pain can be observed, fever if hemorrhage, and/or necrosis, as well as secondary symptoms (e.g., weight loss, anorexia, etc. $)^{[3]}$. 
Laboratory findings are usually not specific (i.e., normal liver tests and negative neoplastic markers) ${ }^{[3]}$. Radiological diagnosis might be difficult because of overlapping features with other pediatric liver masses; ultrasound may show a mass with mixed solid and cystic components and computed tomography usually depicts a large hypodense mass with multiple septations and solid nodules ${ }^{[3-5]}$. The cystic appearance might be misleading, causing delayed management ${ }^{[3]}$. UESL is often larger than $10 \mathrm{~cm}$ at diagnosis, with $75 \%$ of the cases located in the right liver lobe, $10 \%$ in the left lobe, and $15 \%$ in both lobes ${ }^{[3,6]}$. Pathological examination and immunohistochemical analysis of biopsy specimens, as well as of the surgical resection material, is the mainstay of diagnosis for UESL ${ }^{[7]}$. In contrary to other liver tumors such as hepatoblastoma and hepatocellular carcinoma, there is no universally agreed treatment of UESL. But two elements are constant and acknowledged within the worldwide used protocols: UESL is a chemo-sensitive tumor and complete surgical resection is of utmost importance. The aim of this perspective is to discuss the state-of-the-art surgical strategy of UESL as well as the necessity to define a new risk stratification grouping system for this particular pediatric liver malignancy.

\section{TREATMENT PROTOCOLS}

Treatment for UESL evolved from a surgery-only based treatment in the early 1980's, when chemo- and radiotherapy was used only occasionally, to a multimodal treatment management plan including (neo)adjuvant chemotherapy ${ }^{[8]}$. But still, and as mentioned above, the management for UESL varies within different oncological societies, as further addressed below [Table 1]. Nonetheless, the general agreement is as follows: if the mass is resectable, an upfront surgical resection followed by adjuvant chemotherapy is advised; however, if the mass is deemed an unresectable mass, a neoadjuvant chemotherapy, reassessment, surgical resection or liver transplantation, and subsequent chemotherapy is recommended [Figure 1 $]^{[9,10]}$.

As for the timing of surgery after neoadjuvant chemotherapy within the different protocols of the different societies, the "European" treatment of UESL initially, between 1995 and 2005, followed the rules of the Socié té Internationale d'Oncologie Pédiatrique (SIOP) using the "Malignant Mesenchymal Tumors Protocol SIOP-MMT95"; from 2005, the protocol has changed to the RMS2005 EpSSG that evaluates surgical feasibility after the 3 rd course and actually performs it after the 4 th course ${ }^{[9]}$. The German CWS (Cooperative Weichteilsarkom Studie) also evaluates treatment response after 3 courses of chemotherapy, and if deemed feasible a definitive delayed surgery is performed after the 4th course; within both protocols, abdominal irradiation is recommended only if local control cannot be achieved otherwise ${ }^{[11]}$. The American Children Oncology Group (COG) uses different protocols; within the "soft tissue sarcoma protocol", 5 courses of neoadjuvant chemotherapy are employed ${ }^{[10]}$.

As for radiotherapy, the role and average dose in UESL is not well-defined ${ }^{[11]}$. Radiotherapy might help in preventing recurrence but its effectiveness as well as impact on survival is unknown yet ${ }^{[12]}$. Reports of its use in peritoneal dissemination confirm its feasibility but data is still scarce ${ }^{[13]}$. But still, the use of radiation therapy is reported to be as high as in $15 \%$ of the patients ${ }^{[12,14]}$.

A case series dating from 2000, evaluating the role of transhepatic arterial chemoembolization (TACE) in liver tumors, showed that in two children with UESL TACE did not show any response to this type of therapy ${ }^{[15]}$. However, in a recent report of two cases, TACE combined with neoadjuvant chemotherapy with reduced dosage, allowed for significant reduction of tumor size. Zhao et al. ${ }^{[16]}$ consider that TACE might be used as a preoperative therapy in pediatric UESL where the tumor is initially deemed unresectable.

Unresectable tumors might benefit from the transarterial radioembolization as a bridge to resection or transplantation or as a less toxic, palliative treatment alternative ${ }^{[17]}$. Minimal data exists for this treatment 
Table 1. Treatment protocols for paediatric undifferentiated embryonal sarcoma of the liver (UESL)

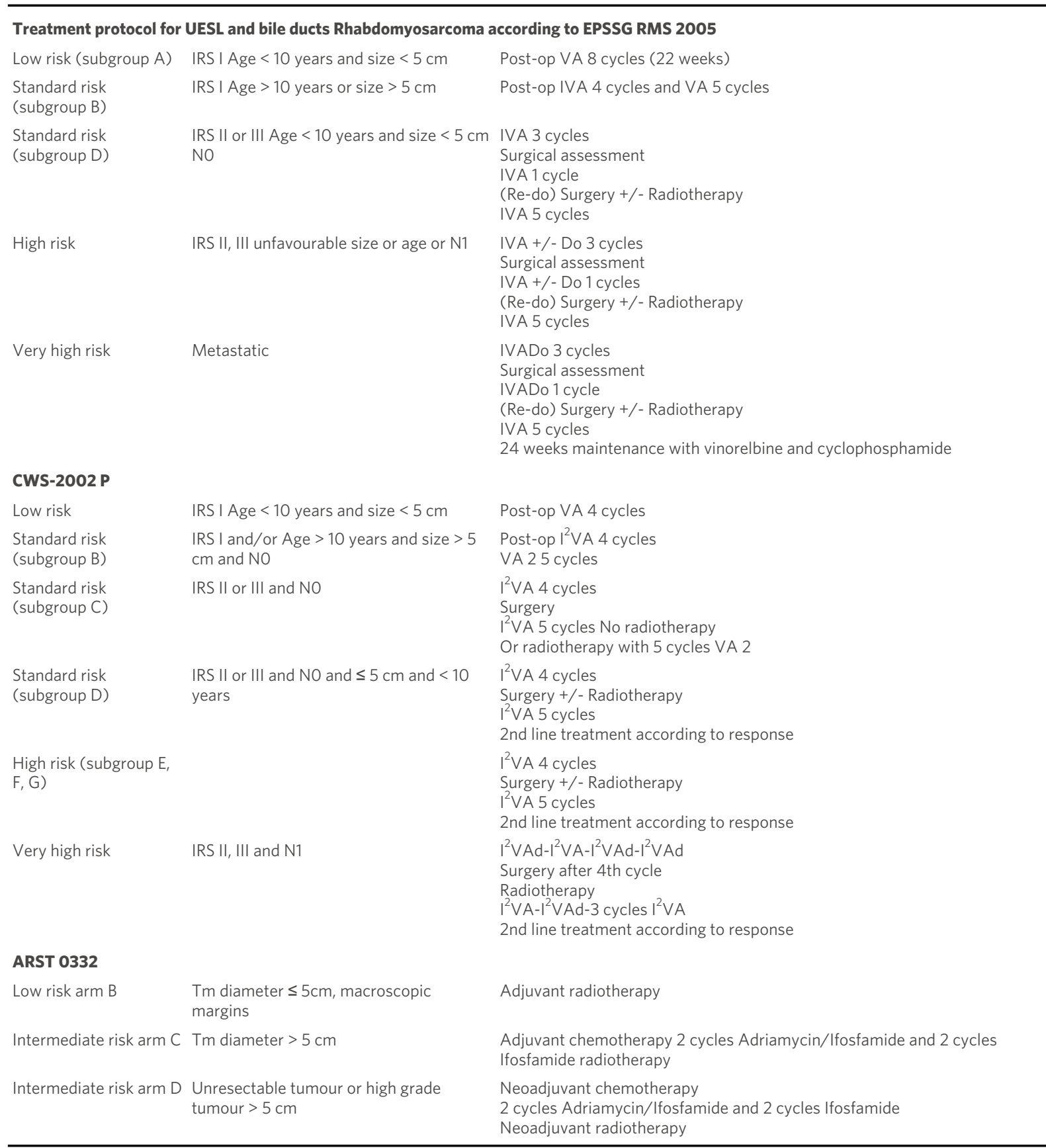

UESL: Undifferentiated embryonal sarcoma of the liver; IRS: intergroup rhabdomyosarcoma study; IVA: ifosfamide, vincristine, actinomycin D; IVADo: ifosfamide, vincristine, actinomycin D, doxorubicin; VA: vincristine, actinomycin D; Source: clinicaltrial.gov (id: NCT00379457).

option in pediatric liver malignancies ${ }^{[17,18]}$.

As with other liver tumors, in the case of tumor rupture or spontaneous bleeding and if the patient is unstable, arterial embolization or, if feasible, complete upfront resection might be performed ${ }^{[9]}$. 


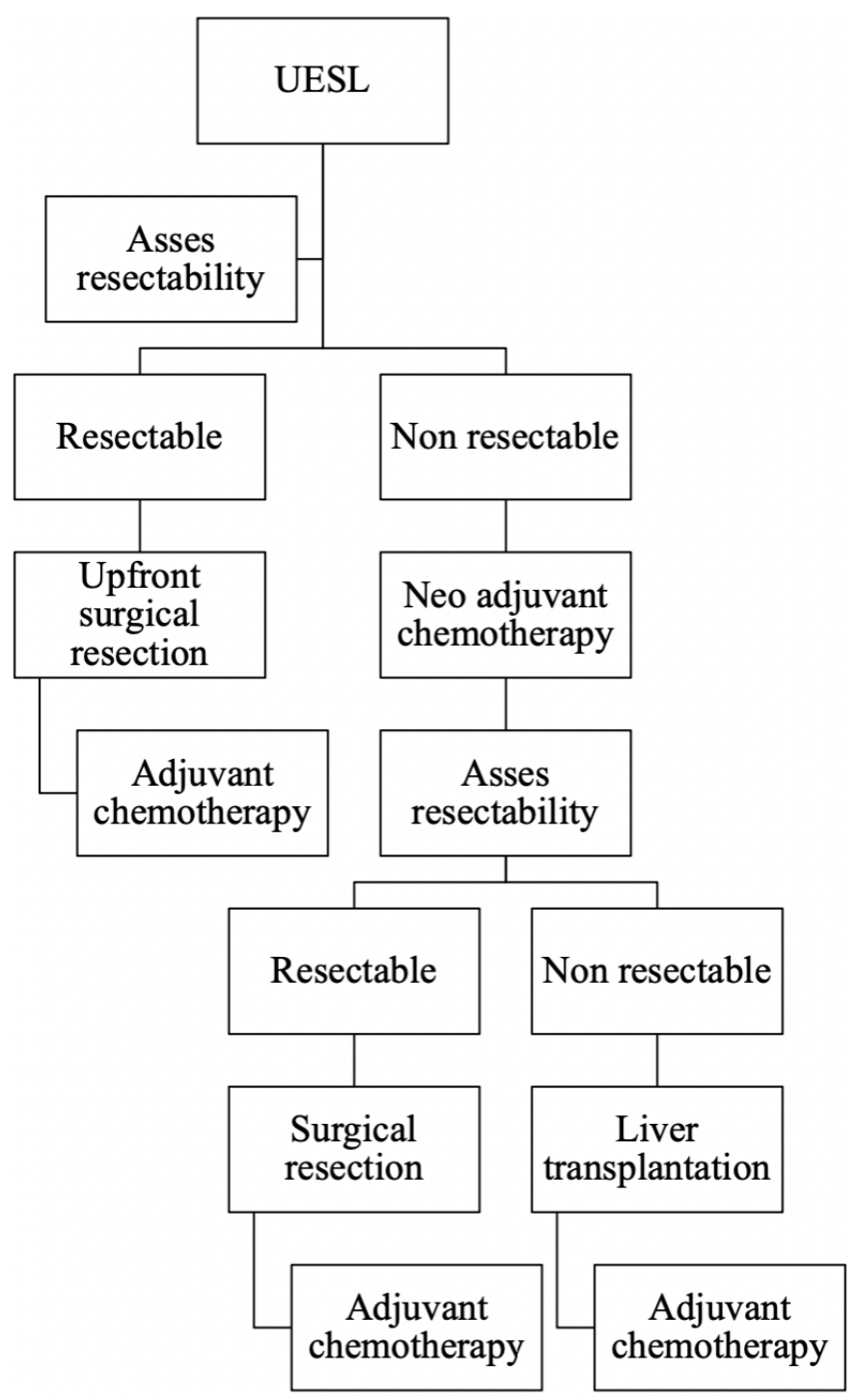

Figure 1. Decisional algorithm for the management of undifferentiated embryonal sarcoma of the liver (UESL).

\section{THE ROLE OF PREOPERATIVE CHEMOTHERAPY}

Preoperative chemotherapy has shown to reduce tumor size and minimizes the risk of intraoperative spillage or microscopic residue. Between $30 \%$ to $40 \%$ of the patients might not be amenable to a primary tumor resection and are subjected to neoadjuvant chemotherapy to facilitate a complete surgical resection ${ }^{[1,14]}$. In fact, after neoadjuvant chemotherapy, resectability has been reported to improve in up to $81 \%$ in recent series, with varied regimens that are basically used for other pediatric soft tissue sarcomas ${ }^{[1,119]}$. Another, less studied effect of the neoadjuvant chemotherapy is the percentage of tumor necrosis, which ranged between $70 \%-100 \%$ in a series of seven patients, even if the radiologic response was not as obvious as the pathologic response ${ }^{[9]}$.

Despite the commonly recognized credo that in case of resectable UESL, surgery could or should be done upfront without neoadjuvant chemotherapy, some centers advocate preoperative chemotherapy regardless of the stage of resectability. Resections seem to become easier after chemotherapy, and thus are associated with a reduced morbidity. Nevertheless chemotherapy is also associated with multiple side effects: $5 \%$ and 
$8 \%$ after IRS III regimens 38 and 35, respectively, where two thirds of the complications were reported to be secondary to sepsis developed after neutropenia ${ }^{[20]}$; cardiac toxicity, metabolic complications, respiratory distress syndrome and central nervous system toxicity were less frequently described. Of note, within the EpSSG protocol the number of chemotherapy cycles remains the same even if upfront surgery is performed, which supports the safer attitude of preoperative chemotherapy ${ }^{[9]}$. Yet, there is not enough solid evidence to support the benefit of systematic neoadjuvant chemotherapy versus upfront surgery and larger prospective, multicentric studies are required to draw a conclusion.

\section{SURGICAL STRATEGIES}

The surgical challenges in the treatment of UESL are mainly related to the large size at the moment of diagnosis ${ }^{[10]}$. Fortunately, initial aggressive surgeries are less required nowadays, since, as stated above, neoadjuvant chemotherapy decreases tumor size and makes it more amenable to a more standard complete resection $^{[20,21]}$. The most frequently performed surgical resections are hemihepatectomy (37\%), followed by sectionectomy $(28 \%)$ and trisectionectomy $(10 \%)^{[12]}$. But to allow for proper patient management (i.e., surgery planning), a preoperative staging system is needed.

\section{UESL staging system}

Regrettably, preoperative staging such as COG-staging or PRETEXT is not always mentioned in the literature $^{[12]}$, making interpretations and comparison of publications more difficult. One of the first series reporting on UESL used Intergroup Rhabdomyosarcoma Study clinical grouping [Table 2] ${ }^{[22]}$. In a series of 5 patients, the American COG stage was reported as stage I in 4 patients and stage II in 1 patient (preoperative rupture); no correlations could be found with this tumor staging given the small sample $\operatorname{size}^{[23]}$. PRETEXT staging was used for the UESL in only two series, a review of pediatric liver malignancies in Finland with 7 PRETEXT II-III tumors and a monocentric retrospective review of 6 PRETEXT II-III tumors. Again, given the small sample size, conclusions for tumor size correlation with survival were difficult to draw ${ }^{[1,24]}$. Finally, European prognosis groups are stratified according to the International Rhabdomyosarcoma Study group ${ }^{[0]}$. Given the heterogeneity of staging for UESL, no clear correlations between extent of disease and survival can be made. A uniform reporting with the PRETEXT grouping system might be helpful for the standardization of patient grouping, and subsequent patient management, and thus survival analysis. Additional data including age and size of the tumor, together with the PRETEXT grouping system, could be included in a new risk stratification protocol for UESL.

\section{Positive resection margins}

The significance of microscopic residuum is still debated. In a cohort of 103 patients of which 20 had positive microscopic margins, R1 resection did not seem to affect outcome, $(P=0.11)$, but the best survival rates are still reported for patients with negative margins 95\% 5-year survival vs. 83\% 5-year survival for patients with positive margins (i.e., Ro resection) ${ }^{[1,12,23]}$. Nevertheless, the burden of treatment could not be evaluated in the 103 patient cohort of the American National Cancer Database ${ }^{[12]}$, but consisted in 52\% neoadjuvant chemotherapy in a smaller CWS cohort of 25 patients in which resection type impacted survival $^{[11]}$. Again, larger, standardized studies focusing on the matter are needed to obtain a definite answer on the effect of microscopic margins on outcome.

\section{Metastatic UESL}

Although UESL metastases are seen in only $15 \%$ of cases $^{[12]}$, overall survival falls from $91 \%$ in patients without metastatic disease to $70 \%$ in patients with metastatic UESL ${ }^{[12]}$. The most common sites for metastasis are the lungs, adrenal gland, peritoneum, and pleura ${ }^{[12,25-28]}$. Cardiac metastases are found in 10\%$25 \%$ of the patients dying from UESL. Surgical approach in this situation is tailored to the tumor type and extension; staged approaches are reported: either removal of cardiac extension under cardiopulmonary 
Table 2. PRETEXT, COG and IRS liver tumor staging systems

\begin{tabular}{ll}
\hline PRETEXT & \\
PRETEXT I & 3 contiguous sections tumour free \\
PRETEXT II & 2 contiguous sections tumour free \\
PRETEXT III & 1 contiguous section tumour free \\
PRETEXT IV & No contiguous section tumour free \\
COG staging system & \\
Stage I & Complete gross resection at diagnosis with clear margins \\
Stage II & Complete gross resection at diagnosis with microscopic residual disease at the margins of resection \\
Stage III & Biopsy only at diagnosis or gross total resection with nodal involvement or tumor spill or incomplete resection \\
Stage IV & with gross intra-hepatic disease \\
IRS clinical grouping & Metastatic disease at diagnosis \\
Group I & \\
Group II & Localized disease, completely resected \\
Group III & Macroscopic complete but microscopic residual \\
Group IV & Macroscopic residual disease \\
\hline
\end{tabular}

PRETEXT: Pre Treatment Extent of Disease; COG: children's oncology group; IRS: intergroup rhabdomyosarcoma study.

bypass followed later by hepatic tumor resection, or the opposite, hepatic tumor resection first, followed by removal of cardiac extension under cardiopulmonary bypass ${ }^{[2,30]}$.

\section{Preoperative tumor rupture}

Preoperative tumor rupture raises questions about recurrence as it can lead to inappropriate primary tumor resection ${ }^{[9]}$. Spontaneous tumor rupture was reported to occur in $6.5 \%$ of the patients ${ }^{[31]}$. The emergency treatment in case of spontaneous or post biopsy tumor rupture is arterial embolization of the feeding $\operatorname{artery}^{[32,33]}$. Recent data supports that secondary tumor resection with preoperative chemotherapy is more successful than primary resection without an effective adjuvant regimen in this particular setting ${ }^{[31]}$.

\section{Unresectable UESL and liver transplantation}

In the context of unresectable tumors [Table 3] ${ }^{[20]}$, two strategies might be adopted and can be defended, aggressive resections versus liver transplantation as an alternative ${ }^{[20]}$. A recent review reported on twelve liver transplanted patients within the United Network for Organ Sharing, with only one needing a retransplantation and another one succumbing the disease ${ }^{[14]}$. Individual case reports are supporting this increasingly accepted indication ${ }^{[10,14,34-37]}$. Overall, transplantation seems to concern $10 \%$ of UESL patients ${ }^{[12]}$.

\section{COMPLICATIONS AFTER UESL SURGICAL TREATMENT}

Complications after surgical management of UESL are rarely reported, but exist as in all liver surgery. Typically, biliary complications were the most frequently reported complication. In a series of seven patients, bile leaks occurred in $28 \%$ (2/7) of patients, with one resolving spontaneously and one needing an endoscopic retrograde cholangiopancreatography with biliary drainag ${ }^{[22]}$. Biloma rate was higher in a fivepatient case series, in which two (right lobectomy) patients required a drain placement, and one (left lobectomy) a cholecystectomy with bile duct ligation; this last patient also had a cavernomatous transformation of the portal vein ${ }^{[38]}$. A bile duct injury after a right hepatectomy needed a redo surgery with a Roux-en-Y loop ${ }^{[9]}$. 
Table 3. Reported criteria for unresectable UESL

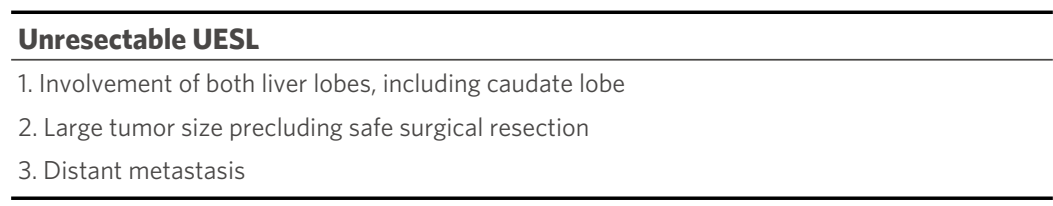

UESL: Undifferentiated embryonal sarcoma of the liver.

Table 4. Possible determinants for the long-term outcome of UESL

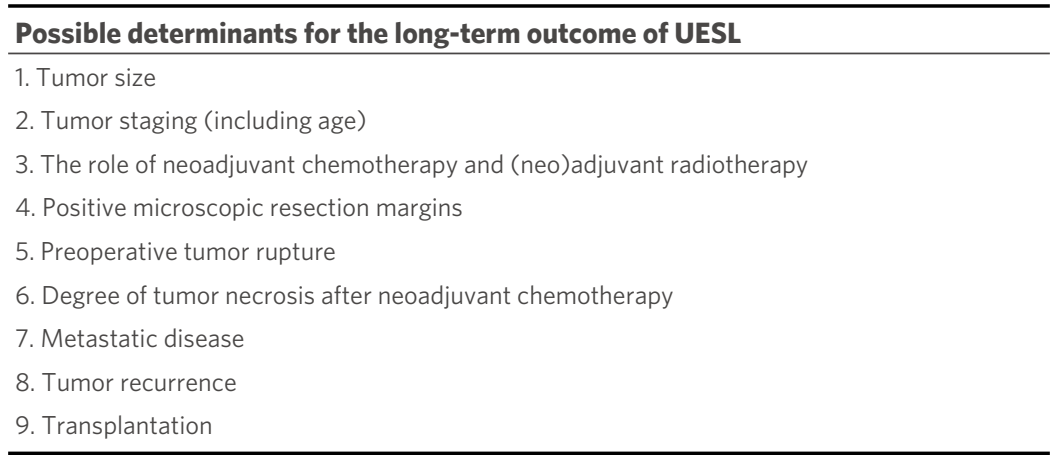

UESL: Undifferentiated embryonal sarcoma of the liver.

Liver failure was described in a three-patient case series causing one patient's death, but data concerning tumor location and surgical excision is scarce ${ }^{[39]}$. One post-resection liver failure needed a rescue liver transplantation in another series ${ }^{[0]}$.

One in 17 patients was reported to die after a right lobectomy in an early report of 2002, due to surgical complications $^{[22]}$.

\section{SURVIVAL}

Five-year survival rate was less than $40 \%$ in the early $1980^{\prime} \mathrm{s}^{[11]}$, with current survival rates higher than $80 \%{ }^{[1,1,14,28]}$. It seems that diagnosis errors with inadequate surgical management lead to lower survival rates $^{[40]}$, again, supporting the evidence that more generally accepted standardized protocols are needed.

Studies focus mainly on the chemotherapy regimens when making correlations with survival rates. The role of other determinants for the long-term outcome summarized in Table 4 needs further clarifications ${ }^{[9,12,41]}$.

\section{CONCLUSIONS}

Over the last decades, the outcome of pediatric UESL has improved. To allow for optimal management of UESL, and thus for accurate outcome analysis, systematic, preoperative neoadjuvant chemotherapy should be further defined, as compared to upfront surgery. A new risk stratification grouping system for UESL including the PRETEXT staging, age of patient, and size of the mass might be considered in a prospective study. Further, the role of positive microscopic margins needs to be defined.

\section{DECLARATIONS}

\section{Authors' contributions}

Made substantial contributions to conception and design of the study, performed data analysis and interpretation, finally approved the version to be published and agreed to be accountable for all aspects of the work: Calinescu AM, Wildhaber BE, Guérin F 
Performed the draft: Calinescu AM, Wildhaber BE

Critical revision of the intellectual content: Wildhaber BE, Guérin F

\section{Availability of data and materials}

Not applicable.

\section{Financial support and sponsorship}

None.

\section{Conflicts of interest}

All authors declared that there are no conflicts of interest.

\section{Ethical approval and consent to participate \\ Not applicable.}

\section{Consent for publication}

Not applicable.

\section{Copyright}

(c) The Author(s) 2021.

\section{REFERENCES}

1. Geel JA, Loveland JA, Pitcher GJ, Beale P, Kotzen J, Poole JE. Management of undifferentiated embryonal sarcoma of the liver in children: a case series and management review. S Afr Med J 2013;103:728-31. DOI PubMed

2. Stocker JT, Ishak KG. Undifferentiated (embryonal) sarcoma of the liver: report of 31 cases. Cancer 1978;42:336-48. DOI PubMed

3. Putra J, Ornvold K. Undifferentiated embryonal sarcoma of the liver: a concise review. Arch Pathol Lab Med 2015;139:269-73. DOI PubMed

4. Cao Q, Ye Z, Chen S, Liu N, Li S, Liu F. Undifferentiated embryonal sarcoma of liver: a multi-institutional experience with 9 cases. Int J Clin Exp Pathol 2014;7:8647-56. PubMed PMC

5. Gabor F, Franchi-Abella S, Merli L, Adamsbaum C, Pariente D. Imaging features of undifferentiated embryonal sarcoma of the liver: a series of 15 children. Pediatr Radiol 2016;46:1694-704. DOI PubMed

6. Goncalves-Matoso V, Renella R, Gudinchet F, Schnyder P, Beck-Popovic M, Lepori D. [Undifferentiated sarcoma of the liver: a rare tumor of childhood]. Arch Pediatr 2005;12:1624-6. DOI PubMed

7. Zhang H, Lei L, Zuppan CW, Raza AS. Undifferentiated embryonal sarcoma of the liver with an unusual presentation: case report and review of the literature. J Gastrointest Oncol 2016;7:S100-6. DOI PubMed PMC

8. Shattaf A, Jamil A, Khanani MF, et al. Undifferentiated sarcoma of the liver: a rare pediatric tumor. Ann Saudi Med 2012;32:203-5. DOI PubMed PMC

9. Merli L, Mussini C, Gabor F, et al. Pitfalls in the surgical management of undifferentiated sarcoma of the liver and benefits of preoperative chemotherapy. Eur J Pediatr Surg 2015;25:132-7. DOI PubMed

10. Walther A, Geller J, Coots A, et al. Multimodal therapy including liver transplantation for hepatic undifferentiated embryonal sarcoma. Liver Transpl 2014;20:191-9. DOI PubMed

11. Murawski M, Scheer M, Leuschner I, et al. Undifferentiated sarcoma of the liver: multicenter international experience of the cooperative soft-tissue sarcoma group and polish paediatric solid tumor group. Pediatr Blood Cancer 2020;67:e28598. DOI PubMed

12. Shi Y, Rojas Y, Zhang W, et al. Characteristics and outcomes in children with undifferentiated embryonal sarcoma of the liver: a report from the National Cancer Database. Pediatr Blood Cancer 2017;64:e26272. DOI PubMed PMC

13. Casey DL, Wexler LH, LaQuaglia MP, Meyers PA, Wolden SL. Favorable outcomes after whole abdominopelvic radiation therapy for pediatric and young adult sarcoma. Pediatr Blood Cancer 2014;61:1565-9. DOI PubMed

14. Techavichit P, Masand PM, Himes RW, et al. Undifferentiated Embryonal Sarcoma of the Liver (UESL): a single-center experience and review of the literature. J Pediatr Hematol Oncol 2016;38:261-8. DOI PubMed

15. Malogolowkin MH, Stanley P, Steele DA, Ortega JA. Feasibility and toxicity of chemoembolization for children with liver tumors. $J$ Clin Oncol 2000;18:1279-84. DOI PubMed

16. Zhao X, Xiong Q, Wang J, et al. Preoperative interventional therapy for childhood undifferentiated embryonal liver sarcoma: two retrospective cases from a single center. European J Pediatr Surg Rep 2015;3:90-3. DOI PubMed PMC

17. Aguado A, Ristagno R, Towbin AJ, et al. Transarterial radioembolization with yttrium-90 of unresectable primary hepatic malignancy in children. Pediatr Blood Cancer 2019;66:e27510. DOI PubMed

18. Aguado A, Dunn SP, Averill LW, et al. Successful use of transarterial radioembolization with yttrium-90 (TARE-Y90) in two children 
with hepatoblastoma. Pediatr Blood Cancer 2020;67:e28421. DOI PubMed

19. Ismail H, Dembowska-Baginska B, Broniszczak D, et al. Treatment of undifferentiated embryonal sarcoma of the liver in children-single center experience. J Pediatr Surg 2013;48:2202-6.

20. Kim DY, Kim KH, Jung SE, Lee SC, Park KW, Kim WK. Undifferentiated (embryonal) sarcoma of the liver: combination treatment by surgery and chemotherapy. J Pediatr Surg 2002;37:1419-23. DOI PubMed

21. Lack EE, Schloo BL, Azumi N, Travis WD, Grier HE, Kozakewich HP. Undifferentiated (embryonal) sarcoma of the liver. Clinical and pathologic study of 16 cases with emphasis on immunohistochemical features. Am J Surg Pathol 1991;15:1-16. PubMed

22. Bisogno G, Pilz T, Perilongo G, et al. Undifferentiated sarcoma of the liver in childhood: a curable disease. Cancer 2002;94:252-7. DOI PubMed

23. Mathias MD, Ambati SR, Chou AJ, et al. A single-center experience with undifferentiated embryonal sarcoma of the liver. Pediatr Blood Cancer 2016;63:2246-8. DOI PubMed PMC

24. Tiusanen T, Hukkinen M, Leskinen O, et al. Incidence and long-term outcomes of surgically treated childhood hepatic malignancies in Finland. Acta Paediatr 2020;109:404-14. DOI PubMed

25. Xie S, Wu X, Zhang G, et al. Remarkable regression of a lung recurrence from an undifferentiated embryonal sarcoma of the liver treated with a DC vaccine combined with immune cells: a case report. Cell Immunol 2014;290:185-9. DOI PubMed

26. Lee MK, Kwon CG, Hwang KH, et al. F-18 FDG PET/CT findings in a case of undifferentiated embryonal sarcoma of the liver with lung and adrenal gland metastasis in a child. Clin Nucl Med 2009;34:107-8. DOI PubMed

27. Plant AS, Busuttil RW, Rana A, Nelson SD, Auerbach M, Federman NC. A single-institution retrospective cases series of childhood undifferentiated embryonal liver sarcoma (UELS): success of combined therapy and the use of orthotopic liver transplant. J Pediatr Hematol Oncol 2013;35:451-5. DOI PubMed

28. Shi M, Xu H, Sangster GP, Gu X. Pulmonary metastases from an undifferentiated embryonal sarcoma of the liver: a case report and review. Case Rep Oncol Med 2018;2018:7840865. DOI PubMed PMC

29. Webber EM, Morrison KB, Pritchard SL, Sorensen PH. Undifferentiated embryonal sarcoma of the liver: results of clinical management in one center. J Pediatr Surg 1999;34:1641-4. DOI PubMed

30. Dominguez-Massa C, Serrano-Martinez F, Blanco-Herrera OR, Berbel-Bonillo A, Hornero-Sos F, Montero-Argudo JA. Treatment of hepatic embryonal undifferentiated sarcoma with cardiothoracic involvement. World J Pediatr Congenit Heart Surg 2020;11:NP199202. DOI PubMed

31. Uchiyama M, Iwafuchi M, Yagi M, et al. Treatment of ruptured undifferentiated sarcoma of the liver in children: a report of two cases and review of the literature. J Hepatobiliary Pancreat Surg 2001;8:87-91. DOI PubMed

32. Yedibela S, Reck T, Ott R, Muller V, Papadopoulos T, Hohenberger W. [Undifferentiated, embryonal sarcoma as a rare cause of spontaneous liver rupture in adults]. Chirurg 2000;71:101-5. DOI PubMed

33. Kupeli S, Yalcin B, Cil BE, Akcoren Z, Buyukpamukcu M. Undifferentiated embryonal sarcoma of the liver in a child complicated by haemorrhage. Pediatr Radiol 2008;38:1259-61. DOI PubMed

34. Schluckebier D, McLin VA, Kanavaki I, Ansari M, Wildhaber BE. The role of liver transplantation in undifferentiated embryonal sarcoma of the liver in children. J Pediatr Hematol Oncol 2016;38:495-6. DOI PubMed

35. Okajima H, Ohya Y, Lee KJ, et al. Management of undifferentiated sarcoma of the liver including living donor liver transplantation as a backup procedure. J Pediatr Surg 2009;44:e33-8. DOI PubMed

36. Kelly MJ, Martin L, Alonso M, Altura RA. Liver transplant for relapsed undifferentiated embryonal sarcoma in a young child. $J$ Pediatr Surg 2009;44:e1-3. DOI PubMed

37. Dower NA, Smith LJ. Liver transplantation for malignant liver tumors in children. Med Pediatr Oncol 2000;34:136-40. DOI PubMed

38. May LT, Wang M, Albano E, Garrington T, Dishop M, Macy ME. Undifferentiated sarcoma of the liver: a single institution experience using a uniform treatment approach. J Pediatr Hematol Oncol 2012;34:e114-6. DOI PubMed PMC

39. Wei ZG, Tang LF, Chen ZM, Tang HF, Li MJ. Childhood undifferentiated embryonal liver sarcoma: clinical features and immunohistochemistry analysis. J Pediatr Surg 2008;43:1912-9. DOI PubMed

40. Wildhaber BE, Montaruli E, Guerin F, Branchereau S, Martelli H, Gauthier F. Mesenchymal hamartoma or embryonal sarcoma of the liver in childhood: a difficult diagnosis before complete surgical excision. J Pediatr Surg 2014;49:1372-7. DOI PubMed

41. Hu HM, Zhang WL, Li J, et al. Report of seven children with undifferentiated embryonal sarcoma of the liver. Chin Med J (Engl) 2019;132:2244-5. DOI PubMed PMC 